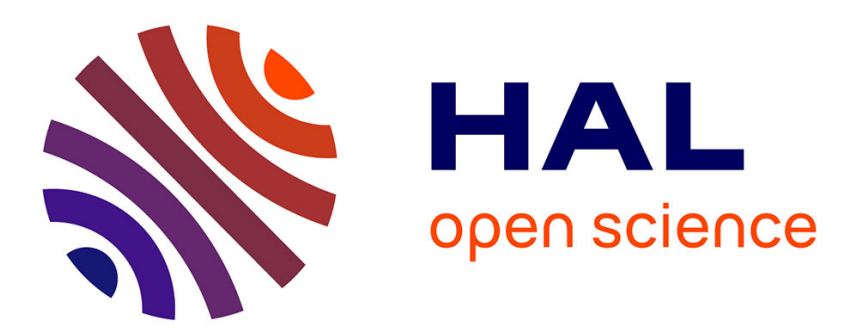

\title{
Micromachined array-type Mirau interferometer for parallel inspection of MEMS
}

J. Albero, S. Bargiel, N. Passilly, P. Dannberg, M. Stumpf, U.D. Zeitner, C. Rousselot, K. Gastinger, C. Gorecki

\section{- To cite this version:}

J. Albero, S. Bargiel, N. Passilly, P. Dannberg, M. Stumpf, et al.. Micromachined array-type Mirau interferometer for parallel inspection of MEMS. Journal of Micromechanics and Microengineering, 2011, 21 (6), pp.065005. 10.1088/0960-1317/21/6/065005 . hal-00655057

\section{HAL Id: hal-00655057 https://hal.science/hal-00655057}

Submitted on 4 May 2021

HAL is a multi-disciplinary open access archive for the deposit and dissemination of scientific research documents, whether they are published or not. The documents may come from teaching and research institutions in France or abroad, or from public or private research centers.
L'archive ouverte pluridisciplinaire HAL, est destinée au dépôt et à la diffusion de documents scientifiques de niveau recherche, publiés ou non, émanant des établissements d'enseignement et de recherche français ou étrangers, des laboratoires publics ou privés. 


\title{
Micromachined array-type Mirau interferometer for parallel inspection of MEMS
}

\author{
J Albero ${ }^{1}$, S Bargiel ${ }^{1}$, N Passilly ${ }^{1}$, P Dannberg ${ }^{2}$, M Stumpf $^{2}$, U D Zeitner ${ }^{2}$, \\ C Rousselot ${ }^{1}, \mathrm{~K}_{\text {Gastinger }}{ }^{3}$ and C Gorecki ${ }^{1}$ \\ ${ }^{1}$ Département MN2S, FEMTO-ST (UMR CNRS 6174), 16 Route de Gray, 25030 Besançon Cedex, \\ France \\ ${ }^{2}$ Fraunhofer IOF, A-Einstein-Str. 7, 07745-Jena, Germany \\ ${ }^{3}$ SINTEF IKT Optical Measurement Systems and Data Analysis, N-7465 Trondheim, Norway \\ E-mail: christophe.gorecki@univ-fcomte.fr.
}

\begin{abstract}
We present the development of an array type of micromachined Mirau interferometers, operating in the regime of low coherence interferometry (LCI) and adapted for massively parallel inspection of MEMS. The system is a combination of free-space micro-optical technologies and silicon micromachining, based on the vertical assembly of two glass wafers. The probing wafer carries an array of refractive microlenses, diffractive gratings to correct chromatic and spherical aberrations and reference micro-mirrors. The semitransparent beam splitter plate is based on the deposition of a dielectric multilayer, sandwiched between two glass wafers. The interferometer matrix is the key element of a novel inspection system aimed to perform parallel inspection of MEMS. The fabricated demonstrator, including $5 \times 5$ channels, allows consequently decreasing the measurement time by a factor of 25. In the following, the details of fabrication processes of the micro-optical components and their assembly are described. The feasibility of the LCI is demonstrated for the measurement of a wafer of MEMS sensors.
\end{abstract}

\section{Introduction}

The potential of non-destructive optical techniques was demonstrated in the determination of local material properties and for the dynamic characteristics study of MEMS. In particular, optical interferometry may be carried out at wafer level without sample preparation, offering high sensitivity, non-contact measurements and automatic analysis of the results for the reconstruction of the exact 3D shape of MEMS structures. Various techniques were developed including conventional or phase-shifting interferometry $[1,2]$, holographic interferometry and digital holography [3], white light stroboscopic interferometry [4], speckle interferometry [5] and electronic speckle pattern interferometry (ESPI) [6].

According to the measuring principle and detection system, each optical technique is mostly specialized in the characterization of static and/or dynamic behavior of microcomponents, measuring the out-of-plane deformation, inplane deformation or both. Thus, two-beam interferometry requires mirror-like surfaces, while ESPI is able to operate with rough surfaces. Commercially available interference microscopes [7] often use two types of interferometric objectives, which are modifications of the Michelson interferometer: the Mirau and Linnik configurations. In a Michelson interferometer objective, suitable for small magnifications, a beamsplitter cube and a reference mirror are inserted between the objective and the sample. At magnifications up to $50 \times$, the working distance becomes too short to squeeze in a beam splitter cube. A Mirau interferometric objective is therefore used instead. The advantage of such a configuration is to place a reference mirror at the center of the objective lens, and interposing 


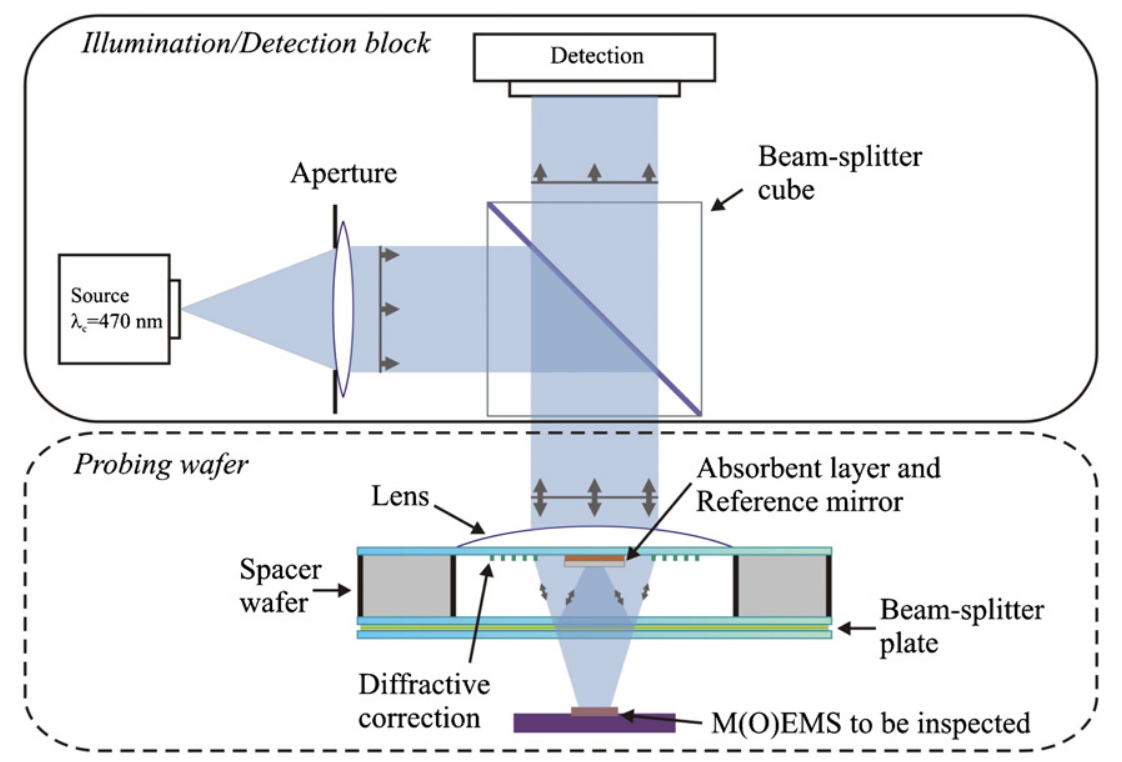

Figure 1. Schematic view of a single-channel LCI. The dashed line encloses the system scope of this paper.

a semi-transparent plate between the objective lens and the specimen. These components are arranged in such a manner that an interference pattern will appear if the system is focused upon the sample. For magnifications of $100 \times$ and more, which require even shorter working distances, a Linnik interferometric objective is used. In the Linnik configuration, a beam splitter cube directs the beam onto two objectives; one beam illuminates the reference mirror and the other is directed to the test surface. However, the vertical and lateral resolutions of a Mirau interferometer are well suited for the analysis of most MEMS devices and its range of working distances is well adapted to the requirements of wafer-level measurements. In this field, most of the metrology architectures are based on bulk microscopes, performing serial measurements. Here, there is a need for miniature and low cost instruments, able to read the data in parallel- this is the proposal of the present contribution.

We will demonstrate that, when fabricated as a combination of free-space micro-optical technology and standard micromachining, an array implementation of miniature Mirau interferometers is well suited to facilitate the in-line metrology of wafer-scale MEMS objects with high throughput. The proof-of-concept of such an approach to MEMS metrology has been demonstrated by the authors [8] and a multifunctional inspection platform with two exchangeable wafer-level sensing heads (called probing wafers) has been developed for MEMS metrology. Each probing wafer contains a network of $5 \times 5$ interferometers where the architecture and the pitch between elementary channels are adapted and aligned on the MEMS wafer to be measured. In addition, the illumination, detection and sample excitation modules can be exchanged in accordance to the category of measurements (topography, dynamic characterization, partially coherent or monochromatic illumination, etc), thanks to their modularity. Two versions of probing wafer configurations are available, each of them being developed over a 4 inch wafer: (i) a network of miniature Mirau interferometers operating in the regime of low coherence interferometry (LCI); and (ii) a network of Twyman-Green laser interferometers (LI). The LCI is dedicated to static measurements of MEMS wafers, whereas the LI is used to characterize dynamic parameters of MEMS. In the final version, the size of each matrix is aimed to reach $10 \times 5$ elements and therefore be adapted when assembled into an 8 inch basis wafer. Each array of interferometers is made to be mounted on commercially available positioning systems, such as the PA 200 prober from Karl Suss [9]. Light sources are arranged in an array and positioned on each side of each interferometric unit. The light is guided by a beam splitter towards the probing wafer.

In this paper, we focus on the implementation of an arraytype micromachined miniature Mirau interferometer, detailing the fabrication process of micro-optical components and their assembly. In section 2 we present the architecture of the LCI and its main parameters. In section 3, we focus on the fabrication of the probing wafer itself, with a discussion on the design and fabrication process of each micro-optical building block as well as on the complete assembly. To check out the imaging quality of the LCI, an infrared sensor test wafer is measured in section 4, demonstrating the experimental validation of the working principles. Finally, conclusions and perspectives are given in section 5 .

\section{LCI architecture}

The architecture of the proposed interferometric system consists of a $5 \times 5$ matrix of single-channel low-coherence interferometers based on a Mirau configuration. In this paper, we will focus, at some points, on the fabrication of a singlechannel interferometer for clarity although the fabrication processes involve the 25 channels systematically. To match several concrete specifications of MEMS/MOEMS metrology 
(a)

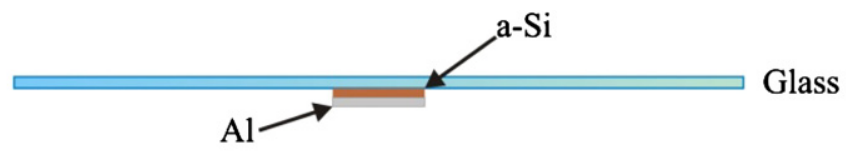

(b)

c)

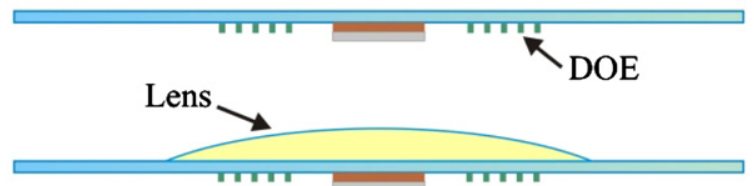

(d)

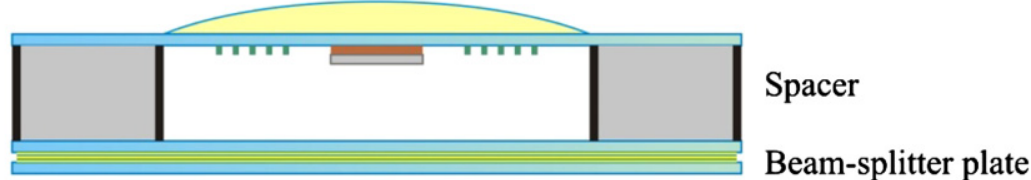

Figure 2. Flowchart of the fabrication process of the interferometer: $(a) \mathrm{Al}$ reference mirrors and the a-Si absorbent layer are created by lift-off, $(b)$ the DOE compensation is fabricated by polymer UV-molding, $(c)$ the microlens is formed by polymer UV-molding and $(d)$ the beam-splitter plate is fabricated by dielectric materials thin-film deposition and assembled by using of a spacer substrate.

in an industrial environment, the matrix is fabricated on a 4-inch basis within a square of nearly $70 \times 70 \mathrm{~mm}^{2}$, where the pitch between the channels is $13.76 \mathrm{~mm} \mathrm{[10].}$ The latter is a multiple of the widespread period in batch fabrication of MEMS systems which allows scanning an entire wafer by small displacements of the wafer within this $13.76 \mathrm{~mm}$ square zone. A schematic view of a singlechannel interferometer is shown in figure 1 . The system includes an illumination/detection block made of a matrix of LED sources operating at a wavelength of $470 \mathrm{~nm}$ and having a $30 \mathrm{~nm}$ spectral width. It is adapted for the needs of a smart-pixel camera, which detects and demodulates the interference signal. The light sources, the beam-splitter cube and the detectors are assembled by mechanical means on the complete architecture of the measurement system. Thus, in the following, we only focus on the fabrication and working demonstration of the probing wafer of micro-optical Mirau interferometers (surrounded by the dashed line in figure 1), without detailing the principles of illumination and detection blocks.

In a single-channel Mirau interferometer, the incident light beam from a LED source, reflected by a cube beamsplitter, is collected by a microlens and directed towards the sample. A diffractive optical element (DOE) is used in order to compensate chromatic and spherical aberrations and therefore to improve the lateral resolution. The collected light passes through a semi-transparent plate, i.e. the beam-splitter plate that reflects half of it back to a reference mirror while the rest of light is transmitted towards the surface to be measured. The beams reflected by the sample and by the reference mirror interfere and the generated interference pattern is directed by the microlens towards the smart-pixel camera module, which detects and demodulates the interference signal [11].

\section{Fabrication of micro-optical building blocks}

The probing system includes four different micro-optical components. The refractive microlens has a spherical shape, a diameter of $2.5 \mathrm{~mm}$ and a sagitta (sag) of $162 \mu \mathrm{m}$ in order to achieve a numerical aperture (NA) of 0.135 (focal length $\approx 9.3 \mathrm{~mm}$ ). The correction of the aberration induced by the microlens is achieved by a DOE placed on the backside of the microlens and presenting a phase function of radial symmetry. The $700 \times 700 \mu \mathrm{m}^{2}$ reference square micromirror is located at the center of the DOE. Finally, the beam-splitter plate, made of a dielectric multi-layer stack, is located at nearly half of the focal length; thanks to spacers whose function is both to adjust the optical path length and to reduce the crosstalk between channels. This system forms the core of the LCI. It can be noted that this assembled multi-wafer stack, where defects such as thickness variations of substrates or possible deformations of wafers will affect the experimental performances, must be as free of stress as possible in order to match the specifications.

Figure 2 shows a schematic flowchart of the complete fabrication process of the probing wafer. First, the aluminum (Al) reference mirrors, whose upper face is absorbent thanks to an amorphous silicon (a-Si) layer, are created by a lift-off process over a glass substrate (figure $2(a)$ ). In the fabrication flow, the replication of the DOE is done prior to the microlens replication due to the much lower polymer thickness, the nearly planar appearance and the lower bowing of the wafer after polymer curing. However, a test replication of the microlenses is performed prior to the DOE fabrication in order to be characterized and accordingly adjust the DOE design. Once the DOE is replicated around the micromirror (figure 2(b)), the microlens is formed by polymer UV-molding (figure $2(c)$ ). Finally, the beam-splitter plate is fabricated by thin-film deposition of dielectric materials and assembled by using a spacer substrate that equalizes the optical path lengths of the reference and the measurement arms of the interferometer (figure 2(d)). All the glass substrates used for the fabrication are Borofloat 33 from Schott [12]. 

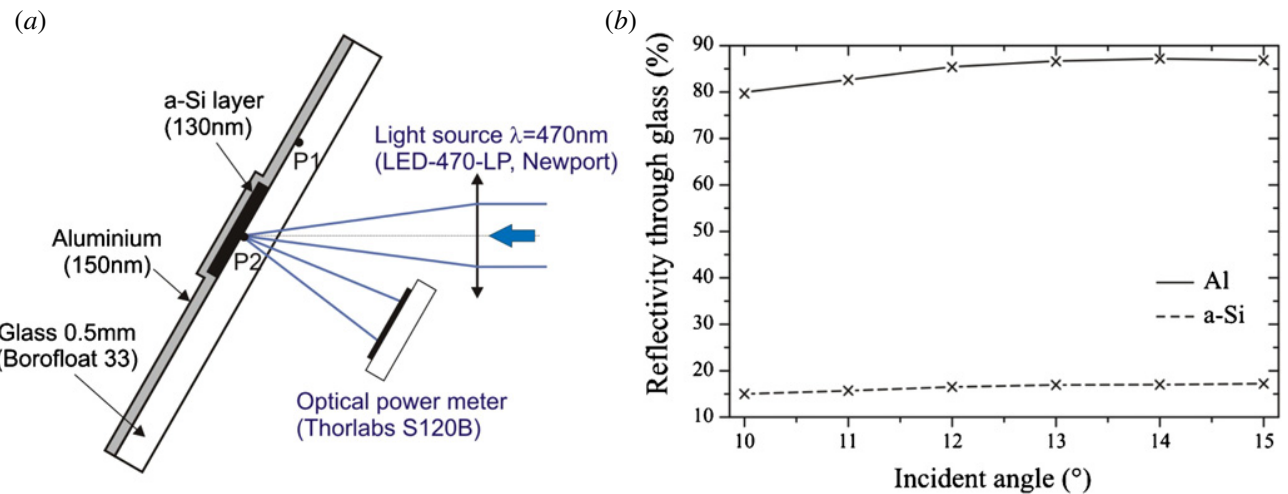

Figure 3. Reflectivity measurements of a-Si/Al micromirrors: (a) setup, (b) comparison between the measured reflectivity of Al (at point $\mathrm{P} 1$ ) and a-Si/Al (at point P2) through the Borofloat 33 substrate as a function of the incident angle of the light beam.

\subsection{Micromirrors}

The function of the micromirrors in the Mirau architecture is to create the reference beam of the interferometer (figure $2(a)$ ). They are made of a thin film of aluminum, deposited on the surface of a $500 \mu \mathrm{m}$ thick glass substrate that eventually receives the microlenses on its opposite side. However, for this architecture, the $\mathrm{Al}$ micromirrors also reflect back the incoming light (through the lens) towards the camera module. These reflections must be reduced as much as possible in order to avoid stray light and thus a contrast reduction of the desired interference image. We have chosen to deposit an amorphous silicon (a-Si) layer between the glass substrate and the $\mathrm{Al}$ layer, because of its absorptive behavior at the wavelength of $\lambda=470 \mathrm{~nm}$ and its good compatibility with the fabrication flowchart.

The $\mathrm{Al}$ and a-Si layers are patterned in a lift-off process. First, a glass substrate is spin-coated with a layer of LOR10B resin (Microchem), specially conceived as the under-layer for positive lift-off processes [13]. Since the LOR is not photosensitive, it is covered with a layer of a positive photoresist S1813 (Shipley). In the standard photolithography process, squares of $700 \times 700 \mu \mathrm{m}^{2}$ are created in $\mathrm{S} 1813$, whereas LOR provides the necessary undercut for lift-off. After patterning the resists, a $130 \mathrm{~nm}$ layer of a-Si is produced by plasma enhanced chemical vapor deposition (PECVD) at a temperature lower than the typical values $\left(80{ }^{\circ} \mathrm{C}\right.$ instead of $360{ }^{\circ} \mathrm{C}$ ) to preserve the resist layers. Afterwards, a $150 \mathrm{~nm}$ thick layer of $\mathrm{Al}$ is evaporated in a highly energetic process (deposition rate $4-5 \mathrm{~nm} \mathrm{~s}^{-1}$ ) specially developed to achieve very low values of surface roughness and, at the same time, to compensate the increased surface roughness caused by the a-Si deposition. Note that the values of average roughness $R_{a}$, obtained using a contact profilometer AlphaStep (Tencor) on top of the Al micromirror are in the order of $0.6 \mathrm{~nm}$, and thus below typical roughness specifications for optical quality components [14]. The glass substrate is then immersed in 1165 remover (Microchem) in order to eliminate the unnecessary metalized zones surrounding the micromirrors. After rinsing and cleaning the surface with an $\mathrm{O}_{2}$ plasma process by reactive ion etching (RIE), the substrate is ready to receive the other micro-optical components.
Table 1. Essential parameters of the micromirrors.

\begin{tabular}{ll}
\hline Parameter & Measured value \\
\hline $\begin{array}{l}\text { Dimensions } \\
\text { Materials }\end{array}$ & $700 \times 700 \mu \mathrm{m}^{2}$ \\
$\begin{array}{l}\text { Layer thickness } \\
\text { Purface roughness }\end{array}$ & $\begin{array}{l}130 \mathrm{~nm} / 150 \mathrm{~nm} \\
\text { Reflectivity of a-Si/Al stack } \\
\text { (direct illumination) }\end{array}$ \\
$\begin{array}{l}\text { Reflectivity of Al (illumination } \\
\text { through glass) }\end{array}$ & $79 \%$ (angle $10^{\circ}, \lambda=470 \mathrm{~nm}$ ) \\
$\begin{array}{l}\text { Reflectivity of a-Si/Al stack } \\
\text { (illumination through glass) }\end{array}$ & $15 \%$ (angle $\left.10^{\circ}, \lambda=470 \mathrm{~nm}\right)$ \\
\hline
\end{tabular}

The characterization of the reflectivity, on the one hand of the Al layer alone, and on the other hand of the a-Si/Al layer stack, when the light (commercial LED source Newport, $\lambda=$ $470 \mathrm{~nm}$ ) is incident through the Borofloat 33 glass substrate, demonstrates the efficiency of the absorbent layer. Indeed, reflectivity measured through the glass substrate is below $17 \%$ for the stack whereas it reaches $85 \%$ without the absorbant layer, corresponding to a reduction of the undesired reflections by a factor 5 (see figure 3 ). The main fabrication parameters and measurement results are listed in table 1.

\subsection{Microlenses}

The polymer microlenses are replicated on top of the glass wafer that contains the micromirrors and the DOEs. As mentioned before, the fabrication of the DOE, used to compensate the aberrations introduced by the imaging microlenses [15], is prior to the replication of the latter. However, since the DOE structure can be adjusted in order to compensate possible deviations of the real geometry of the fabricated microlenses compared to the designed one, the microlens master is fabricated first.

Photolithography and resist reflow are basic technologies in our development for lens mastering, as shown in figure 4 $[16,17]$. First, a clean glass substrate is spin-coated with a layer of Clariant AZ4562 photoresist of submicron thickness homogeneity (figure $4(a)$ ). It can be noted that a thickness of $100 \mu \mathrm{m}$ is sufficient to achieve the needed sag at the end of the process $(\approx 162 \mu \mathrm{m})$. The photoresist is patterned by photolithography and $2.5 \mathrm{~mm}$ diameter cylinders are obtained 
(a)

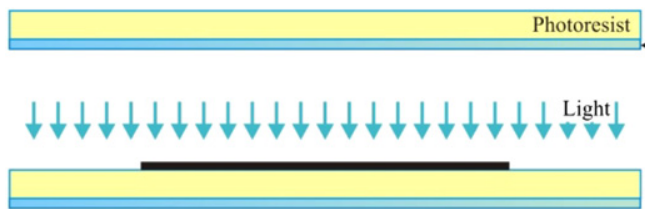

(c)

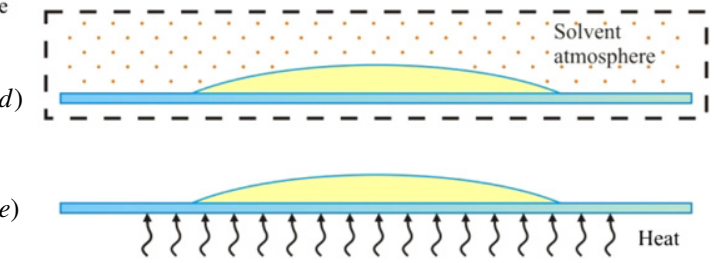

Figure 4. Flowchart of the resist lenses master fabrication: $(a)$ coating of a substrate with photoresist, $(b)$ photolithography of the photoresist, $(c)$ development of the resist to obtain cylinders, $(d)$ cylinders reflow in a solvent atmosphere, and $(e)$ baking of the resulting microlenses.
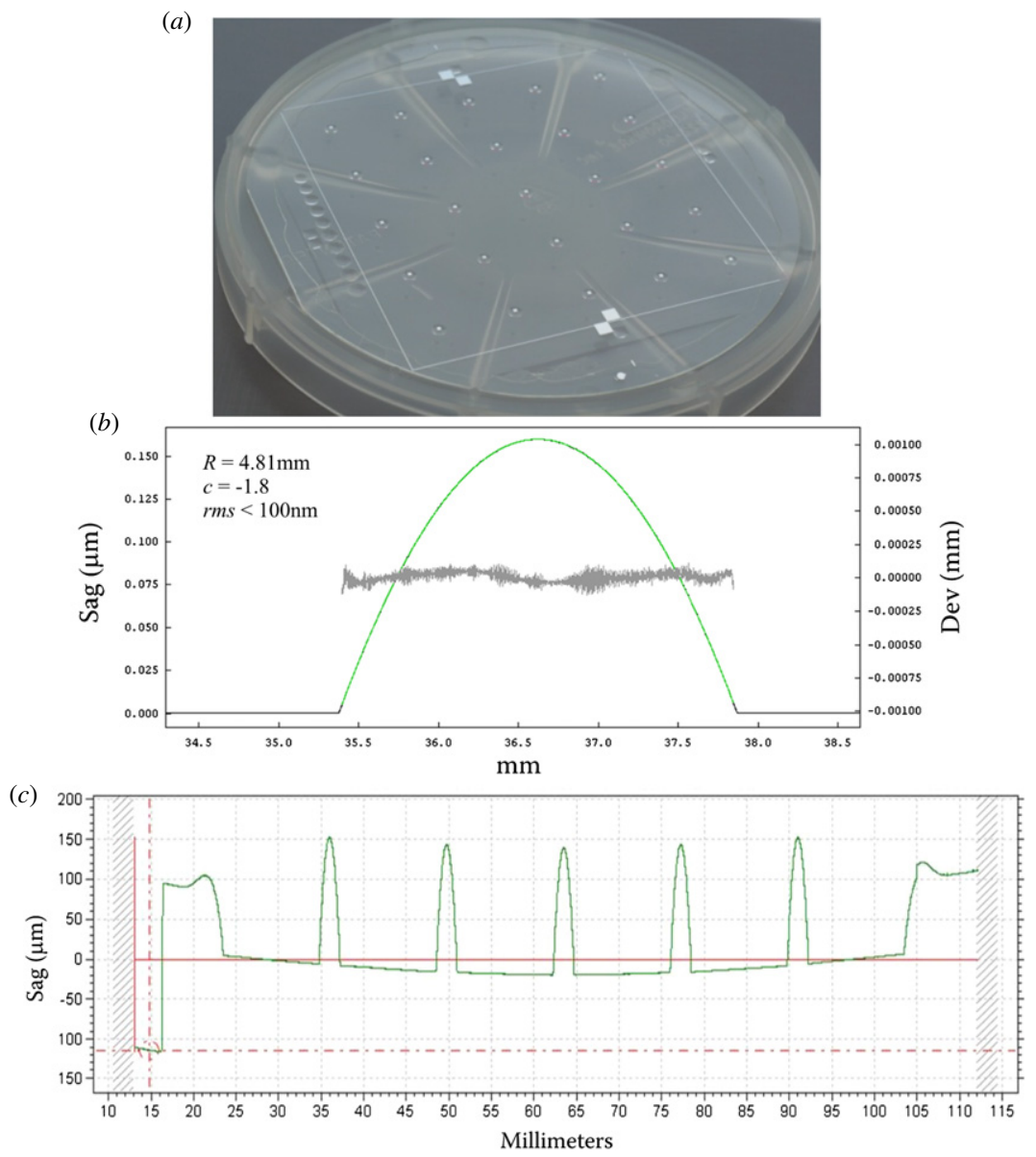

Figure 5. (a) Image of the substrate containing the microlenses and the micromirrors, $(b)$ profile of a single microlens (where $R$ is the radius of curvature, $c$ is the conical constant) and (c) profile of the complete test replication across the sample showing a $20 \mu \mathrm{m}$ bowing of the substrate.

after development (figures $4(b)$ and $(c)$ ). The cylinders are afterwards reflowed in a solvent atmosphere and the surface tension tends to create spherical profiles (figure $4(d)$ ). Finally, the master object is obtained after additional baking (figure $4(e)$ ). In cases of microlens diameters of a few hundred micrometers, this process is well able to produce spherical surfaces. However, when dealing with $2.5 \mathrm{~mm}$ apertures, the weight of the resist leads to its subsidence and the lens profile is not perfectly spherical anymore. An upside down position during the fabrication is therefore preferred, which leads to aspherical profiles with a negative conical constant and thus to lower aberrations and a less challenging DOE compensation.
The obtained resist-based spherical lenses are then replicated in a more resistant material, such as PDMS, in order to create the mold to be used in the operational elements replication. The final microlenses are obtained by UV-molding of ORMOCOMP polymer [18] in a SUSS MicroTec contact mask aligner MA6 with special UV-molding tools and software [19] (figure 5(a)). Sufficient precision and lateral positioning accuracy on the wafer are achieved by using lithographic techniques to generate the structures.

The characterization of the fabricated refractive lenses with a Taylor Hobson Form Talysurf PGI+ profilometer shows minor differences from the lens design. The values are listed in table 2. Nevertheless, the slight error concerning the 


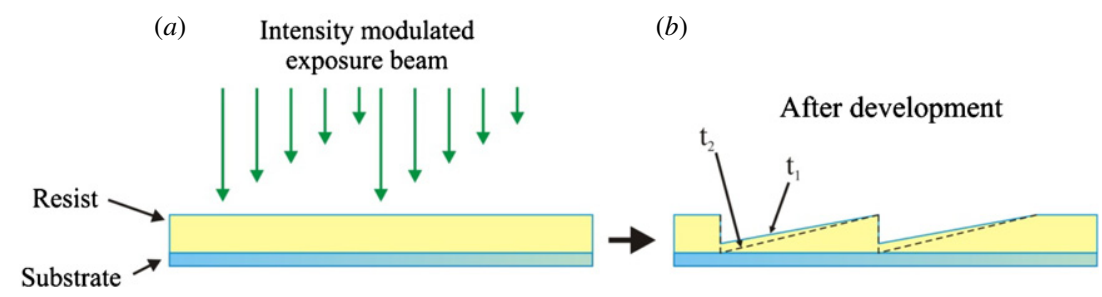

Figure 6. Fabrication scheme of the compensation DOE masters: $(a)$ a photoresist layer is spin-coated over a substrate and exposed to a intensity-modulated laser beam, $(b)$ the photoresist is developed and the depth of the profile depends on the intensity of the beam and the time of development $\left(t_{1}\right.$ and $t_{2}$ being different development times, $\left.t_{1}<t_{2}\right)$.

Table 2. Essential parameters of the lenses.

\begin{tabular}{lll}
\hline Parameter & Measured value & Design value \\
\hline Pitch & $13759.5 \mu \mathrm{m}$ & $13760 \mu \mathrm{m}$ \\
Radius of curvature & $4820 \pm 20 \mu \mathrm{m}$ & $4900 \mu \mathrm{m}$ \\
Conical constant & -1.8 & 1 \\
Form deviation & $<100 \mathrm{~nm}$ & $0 \mathrm{~nm}$ \\
Lens sag & $162 \mu \mathrm{m}$ & $162 \mu \mathrm{m}$ \\
Refractive index & 1.523 & 1.523 \\
(@ $\lambda=530 \mathrm{~nm}$ ) & & \\
Numerical aperture & 0.132 & 0.135 \\
\hline
\end{tabular}

focal length is further compensated by properly adjusting the thickness of the spacer wafer and fine-tuned on each channel independently by setting the exact position of the LED sources of the complete system. The profile of a single lens is reported in figure $5(b)$, where the deviation of the measured profile with respect to the calculated ideal lens for the given conical constant is superimposed. The profile of the complete wafer measured along the $70 \mathrm{~mm}$ of the whole $5 \times 5$ matrix (figure 5(c)) allows estimating the overall bow after the replication. The obtained value of about $20 \mu \mathrm{m}$ is tolerable and is moreover expected to be compensated thanks to the backside replication of the DOEs and by the spacer wafer in the final assembly of the system.

\subsection{Diffractive optical elements}

The diffractive optical elements are conceived to compensate spherical and chromatic aberrations introduced by the planoconvex microlens. They are designed by using the ZEMAX ${ }^{\circledR}$ software. The design has been realized considering a diameter of $2.5 \mathrm{~mm}$ with a non-structured $700 \times 700 \mu \mathrm{m}^{2}$ square at the center to fit the area occupied by the micromirrors. The design takes into account a phase function with radial symmetry defined by

$$
\varphi(r)=a_{2}\left(\frac{r}{r_{o}}\right)^{2}+a_{4}\left(\frac{r}{r_{o}}\right)^{4}
$$

where $a_{2}$ and $a_{4}$ are the values to be optimized and $r_{o}$ is the normalized radius, for which the second term helps reducing the spherical aberrations [20].

The aberration correction diffractive elements are fabricated by variable dose laser lithography in a photoresist layer [21], with a DWL400 FF system from Heidelberg Instruments, as shown in figure 6. First, a clean substrate is spin-coated with a $3 \mu \mathrm{m}$-thick layer of photoresist. This layer is selectively exposed by an intensity-modulated laser beam. The dissolution rate of the resist changes as a function of the exposure dose and, consequently, continuous surface profiles can be generated with a proper control of the energy. The obtained DOE is thus reproduced into a more resistant material, similarly as for lenses, to generate a master. The structures are afterwards replicated over the glass substrate with ORMOCOMP HA497 [18], a hybrid polymer resin with lower viscosity than the one used for the fabrication of the lenses. The use of this hybrid polymer helps improve homogeneity of the layer thickness.

As previously mentioned, the replication of the DOE is done prior to the lens replication. Thus, a test replication of the microlenses was performed in a similar separate substrate in order to characterize the actual values of radius of curvature, conical constant and form deviation. From these characterizations (table 2) and under the assumption that the lenses performance does not change from one replica to another, the parameters of the diffractive correction to be fabricated were optimized to be in agreement with the actual lens profile. The resulting parameters are a depth of $905 \mathrm{~nm}$ and a normalization radius $r_{o}=1.25 \mathrm{~mm}$ with aspherical coefficients $a_{2}=-97.117554$ and $a_{4}=$ -22.32441 .

Figure 7(a) shows an image of one of the DOEs with the $700 \mu \mathrm{m} \times 700 \mu \mathrm{m}$ square mirror at the center. The profiles of the DOE are shown in figures 7(b) and (c), where the polymer thickness across the whole matrix can be seen, as well as the cross section of a single replicated DOE. The height variations at the polymer surface (figure $7(b)$ ) are due to thickness variations of the glass substrates and bowing of the replication tool and mask aligner tooling. They can also be attributed to the residual wedge error between the substrate and the tool during the UV curing step. It must be noted, in figure $7(c)$, that the influence of the stylus profile affects the measurement slightly.

\subsection{Beam-splitter plate}

The last optical component required for the Mirau interferometer is a beam-splitter plate (BSP) whose function is to create the reference beam and, at the same time, to transmit the light toward the sample to be measured. In our integrated system, a planar BSP, conceived to achieve a ratio transmitted light/reflected light of 50/50 under normal incidence, is developed. It is based on the interferences between light reflected and transmitted by multiple thin layers made of 


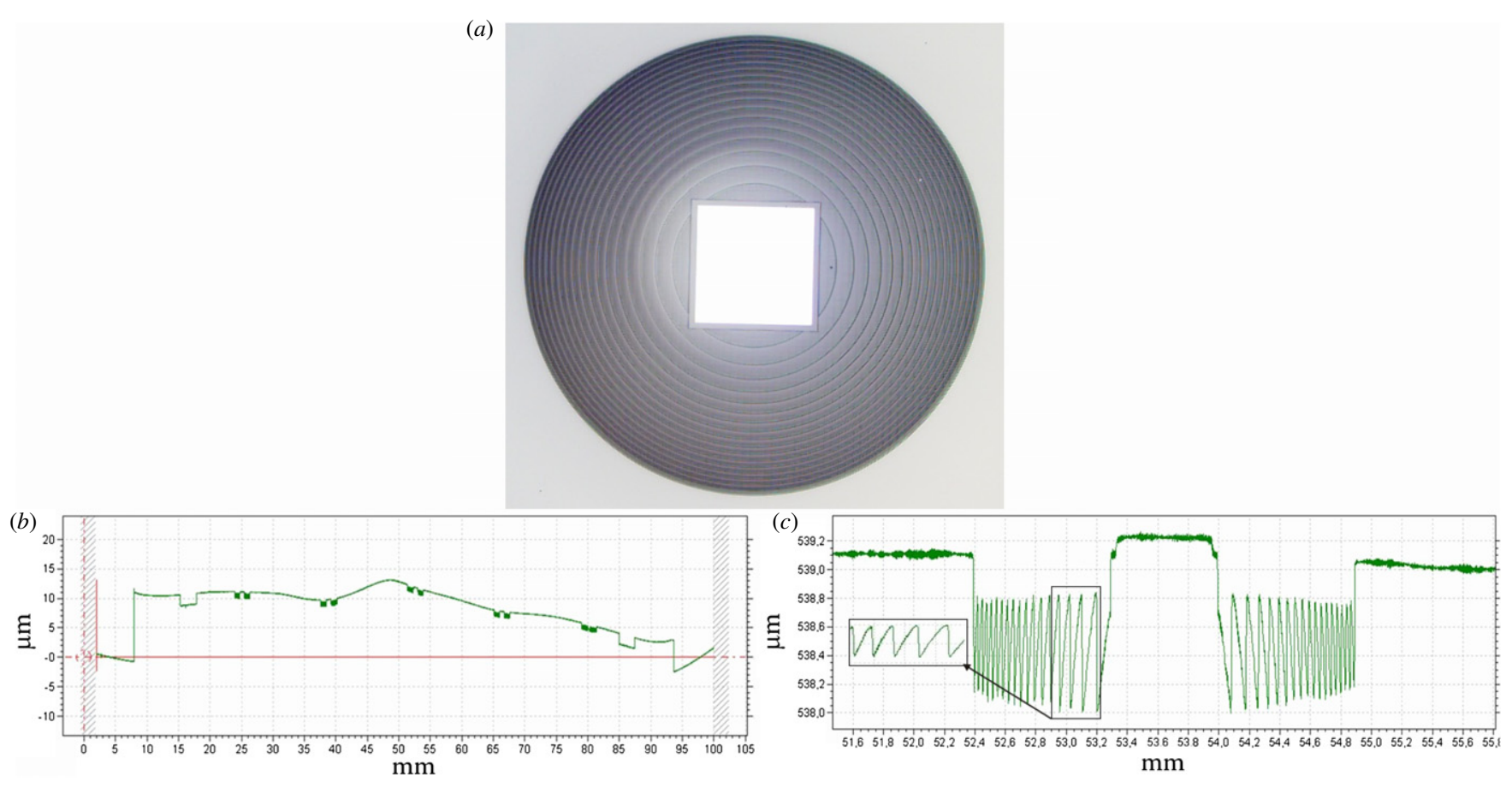

Figure 7. (a) Image of one of the fabricated DOE with the micromirror at the center, $(b)$ profile of the polymer layer thickness across the wafer showing a maximum non-uniformity of approx. $10 \mu \mathrm{m}$, and $(c)$ profile of the cross section of the replicated DOE with a detail of the first four central rings.

Table 3. Design of the beam-splitter plate.

\begin{tabular}{lllll}
\hline & & \multicolumn{3}{c}{ HRI layer } \\
\cline { 4 - 6 } Number of layers & LRI layer thickness $(\mathrm{nm})\left(n_{\mathrm{LRI}}=1.464\right)$ & Refractive index $\left(n_{\mathrm{HRI}}\right)$ & Index tolerance & Thickness $(\mathrm{nm})$ \\
\hline 3 & $80.2 \pm 10.0$ & 2.284 & $2.209<n_{\mathrm{HRI}}<2.371$ & $\Delta h_{\mathrm{HRI}}=51.5 \pm 22.2$ \\
5 & $80.2 \pm 8.7$ & 1.969 & $1.926<n_{\mathrm{HRI}}<2.019$ & $\Delta h_{\mathrm{HRI}}=60.0 \pm 13.7$ \\
7 & $80.2 \pm 7.5$ & 1.829 & $1.798<n_{\mathrm{HRI}}<1.863$ & $\Delta h_{\mathrm{HRI}}=64.5 \pm 9.7$ \\
9 & $80.2 \pm 6.2$ & 1.749 & $1.726<n_{\mathrm{HRI}}<1.775$ & $\Delta h_{\mathrm{HRI}}=67.5 \pm 7.5$ \\
11 & $80.2 \pm 5.5$ & 1.698 & $1.679<n_{\mathrm{HRI}}<1.719$ & $\Delta h_{\mathrm{HRI}}=69.5 \pm 6.5$ \\
\hline
\end{tabular}

dielectric material. Thus, layer thicknesses are chosen such that all the reflections are in phase in order to constructively interfere, i.e., the optical path length in each layer is in the order of a quarter of the wavelength. Then, proper combinations of number of layers and layer refractive indices can lead to a $50 / 50$ BSP. It has to be noted that, in order to equalize the optical path between light reflected and transmitted by the BSP, the dielectric multilayer stack is sandwiched between two similar Borofloat 33 substrates. Nevertheless, in our configuration, the light is not monochromatic and not normally incident. We then investigated (with an in-house numerical code) tolerances to find the best suited layer stack. The design takes into account a blue LED source with a $\lambda_{\text {peak }}=470 \mathrm{~nm}$ and a spectral width FWHM $\Delta \lambda=30 \mathrm{~nm}(455-485 \mathrm{~nm})$ as well as angles of incidence $\theta$ up to $10^{\circ}$ corresponding nearly to the lens numerical aperture of 0.15 . The selected materials for the fabrication were $\mathrm{SiO}_{2}$ (characterized by a 'low' index of refraction $n_{\mathrm{LRI}}=1.464$ [22]) and $\mathrm{SiO}_{x} \mathrm{~N}_{y}$ whose 'high' refractive index value $\left(n_{\mathrm{HRI}}\right)$ can be adjusted depending on its stoichiometry. For different layer stacks made of an odd number of layers (first and last layer made of $\mathrm{SiO}_{\mathrm{x}} \mathrm{N}_{\mathrm{y}}$ ), there are corresponding discrete values of $n_{\mathrm{HRI}}$, in the range (1.72.3), which lead to a 50/50 BSP (reported in table 3).

The ratio of transmission/reflection does not evolve much when considering variations of incidence, i.e. $<1 \%$ for $\theta<$ $10^{\circ}$, whether the stack is made of 3 or 11 layers (no longer true for $\theta>10^{\circ}$ ). However, when considering the wavelength bandwidth (455-485 nm), it appears that a BSP made of 3 layers (corresponding $n_{\mathrm{HRI}}=2.28$ ) is much more 'achromatic' (ratio variation $<1 \%$ ) than a BSP made of 11 layers (for which $n_{\mathrm{HRI}}=1.70$ ) where the ratio can deviate by more than $5 \%$.

In addition to the requirements of the system itself (non-normal incidence, source bandwidth), tolerances on fabrication parameters such as thickness and HRI values are crucial. To evaluate their influence, the ratio deviation was considered up to a maximum of 5\% (e.g., a ratio 55/45). The calculated tolerances (see table 3) show again that a minimum number of layers should be sought, since ranges become narrow when the number of layers exceeds 7 . It can be noted that, for the tolerances calculation, the effects of the deviations of each parameter are considered one by one while the other parameters are fixed. 

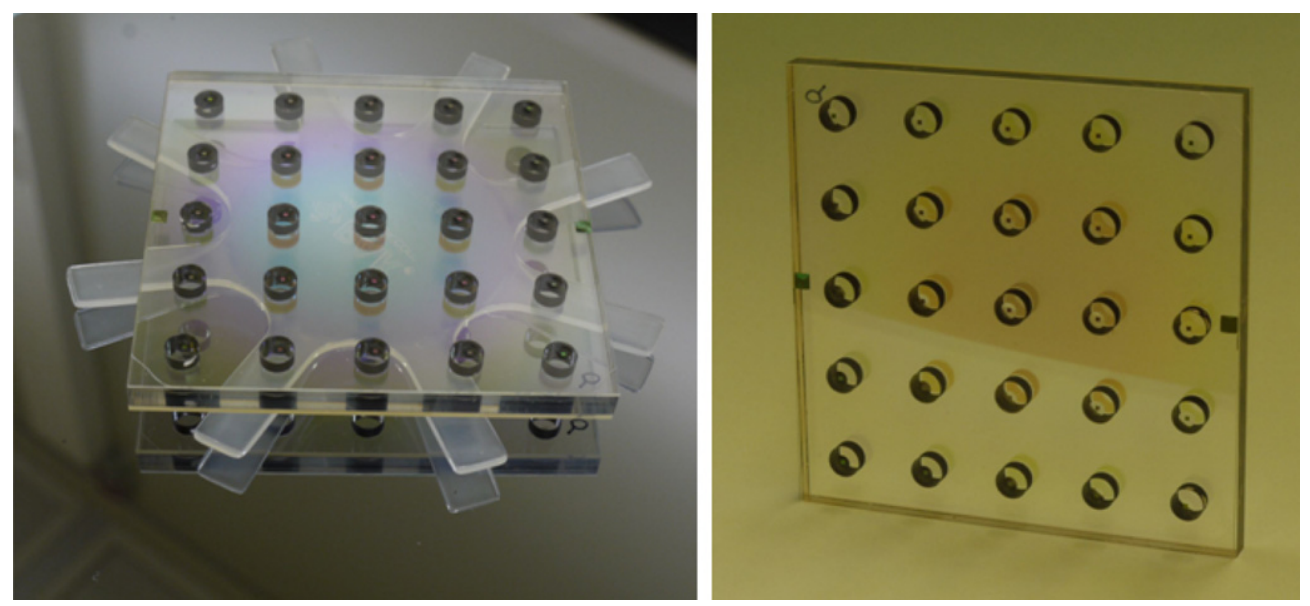

Figure 8. Photographs of the final LCI probing wafer.

Table 4. Main parameters of the dielectric materials deposition.

\begin{tabular}{ll}
\hline Parameter & Value \\
\hline Vacuum limit & $9 \times 10^{-8} \mathrm{mbar}$ \\
Argon flow $\left(Q_{\mathrm{Ar}}\right)$ & 0.75 to $3 \mathrm{sccm}$ \\
RF power & $50 \mathrm{~W}$ \\
Distance target-substrate & $60 \mathrm{~mm}$ \\
Substrate temperature & $20{ }^{\circ} \mathrm{C}$ \\
Oxygen flow $\left(Q_{\mathrm{O} 2}\right)$ & 0.5 to $6 \mathrm{sccm}$ \\
Nitrogen flow $\left(Q_{\mathrm{N} 2}\right)$ & 2 to $6 \mathrm{scc}$ \\
Working pressure & 0.4 to $1.5 \mathrm{~Pa}$ \\
Coating speed $\mathrm{Si}_{x} \mathrm{~N}_{y}\left(\mathrm{~nm} \mathrm{~h}^{-1}\right)$ & 230 to 261 \\
Coating speed $\mathrm{SiO}_{x}\left(\mathrm{~nm} \mathrm{~h}^{-1}\right)$ & to 222 \\
\hline
\end{tabular}

For the fabrication of the BSP, the deposition of each layer is performed by physical vapor deposition (PVD) with an AC450 DC/RF from Alliance Concept, based on a multitarget sputtering system allowing the deposition of inorganic materials over any type of substrate. The films of $\mathrm{SiO}_{2}$ and $\mathrm{SiO}_{x} \mathrm{~N}_{y}$ are deposited by sputtering at radio-frequency $(13.56 \mathrm{MHz})$ in reactive mode, i.e., a controlled flow of reactive gases, $\mathrm{O}_{2}$ or $\mathrm{N}_{2}$, is introduced in the chamber added to argon, the latter being the plasma generator gas. The experimental conditions are described in table 4 . It must be noted that the values listed in table 4 are given as the ranges where the quality of the layers and refraction indices are controlled.

Since the highest values of HRI deduced from numerical calculations were not achievable with $\mathrm{SiO}_{x} \mathrm{~N}_{y}$ material (added to the difficulty of completely venting the chamber and avoiding the presence of oxygen), it was chosen to deposit a five layer stack where the tolerances are still acceptable in terms of fabrication. Such a layer stack was characterized with a Perkin Elmer spectrometer under normal incidence without the upper glass substrate, and the ratio value, once the latter added, is expected to be 55/45, in agreement with the specifications. The deviation from $50 / 50$ ratio can be attributed to the difficult control of the HRI.

\subsection{Final assembly}

In order to finalize the assembly of the probing wafer, the two substrates (one holding lenses, mirrors and diffractive components, the other, the BSP) are separated by a spacer wafer. This allows the adjustment of the optical path between the two arms of the interferometer. Its thickness is adjusted in accordance with the actual performance of the lens and therefore, it is fabricated after its characterization as for the diffractive components. The finally used spacer wafer is made of a $4.2 \mathrm{~mm}$ thick glass block where 25 holes of $4 \mathrm{~mm}$ diameter are drilled in the same matrix configuration as the single channels (pitch $13.76 \mathrm{~mm}$ ). Glass was chosen to achieve high geometrical precision, low thermal expansion coefficient and transparency to facilitate the assembly tasks. The holes were generated by standard ultrasound assisted machining. To avoid cross-talk effects between the different interferometer channels, the hole sidewalls were coated with absorbing black matrix polymer PSK2000 (Brewer Science) which has subsequently been UV-cured and hard baked for $1 \mathrm{~h}$ at $190^{\circ} \mathrm{C}$.

Finally, after dicing each wafer to the final square size of $70 \mathrm{~mm} \times 70 \mathrm{~mm}$ by a wafer saw, they are assembled together by applying UV-curable adhesive on the outer rims. It can be mentioned that this assembly is far from being critical (tolerance over $100 \mu \mathrm{m}$ ) since lenses, DOEs and micromirrors are already aligned during the replication process. Thus, no additional alignment means are required other than aligning the outer borders of the wafers with mechanical edge guides. The assembled probing wafer can be seen in figure 8 .

\section{Experimental validation of LCI}

The experimental setup built to demonstrate the parallel working principle of the interferometer includes five LED sources (Lumiled Rebel from Philips), similar to the ones to be integrated in the complete inspection system, and a standard fire-wire CCD camera with $782 \times 582$ pixels resolution $(A V T$ Guppy F046B). An imaging test was performed prior to the assembly of the lenses substrate with the beam-splitter plate, as seen in figure $9(a)$, which shows the image captured by the CCD camera. The taps in the central ring of the sensor are $10 \mu \mathrm{m}$ wide and their inner details are well resolved, showing a resolution better than $5 \mu \mathrm{m}$. The limits of the square 


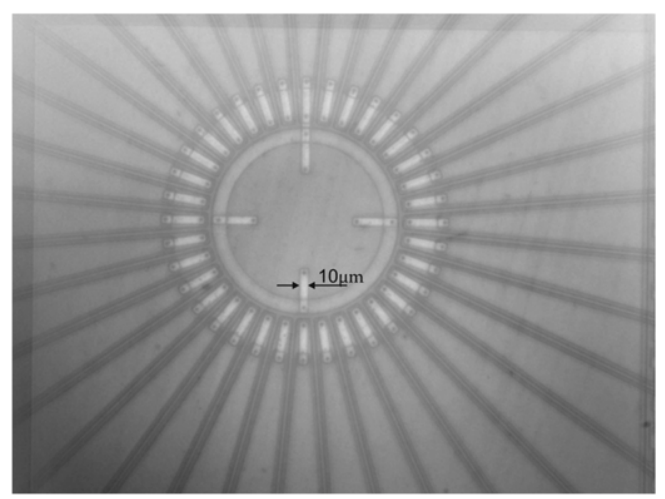

(a)

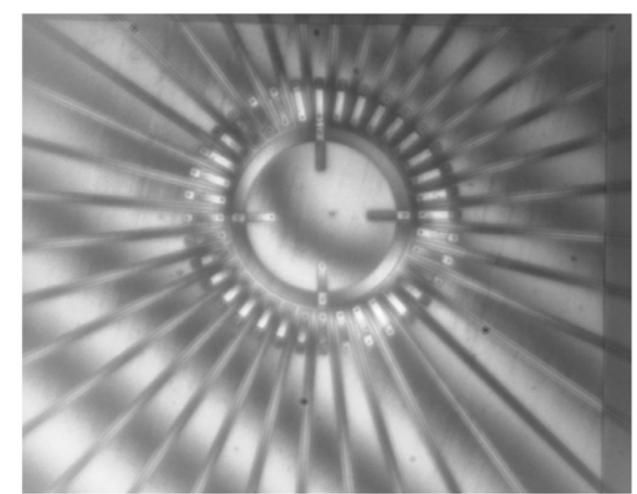

(b)

Figure 9. Optical images of the IR sensor, both formed through the probing wafer: $(a)$ image produced by the lenses with the diffractive correction and the micromirror, before the beam-splitter assembly, and $(b)$ interference fringes over IR sensor created by the complete interferometric system. The square limits of the micromirror are clearly visible on both images.

micromirror, clearly visible, define the field of measurement. When the complete probing wafer was assembled, two tests were performed. First, an optical flat mirror acting as the measured object has been employed to evaluate the flatness within the field of view as well as the systematic measurement error. For all five channels, it results that the flatness value is better than $\lambda / 40$, whereas the systematic error is lower than $\lambda / 20$. Second, a commercially available IR sensor fabricated by Melexis [10] was used as a real MEMS object. Figure $9(b)$ shows how the interference patterns are successfully obtained. The visible fringes show a tilt of the complete structure with respect to the optical axis. The central circle shows circularly formed fringes, showing a concave or convex deformation. The displacement of the fringes over the radial thin structures shows a clear height difference with respect to the main surface. Again, it can be seen on the right-hand side and the top of the image that the useful area of the interferometer is limited by the micromirror size. A USAF 1951 target was used to determine the resolution of the assembled probing wafer, demonstrating a capability to distinguish lines up to $2.4 \mu \mathrm{m}$ wide.

\section{Conclusions and perspectives}

This paper presents the detailed fabrication and experimental validation of a low coherence interferometer probing wafer based on the configuration of array-type Mirau interferometer. We expect that such LCI technology combining the freespace micro-optical technologies and silicon micromachining may have significant impact on the development of parallel inspection of MEMS and MOEMS.

The probing wafer consists of a $5 \times 5$ matrix of single interferometers with a Mirau-based architecture. Each one of them includes four different micro-optical elements, namely lenses, mirrors, diffractive optical elements and a beamsplitter. These elements are fabricated at a wafer level and vertically assembled. The combination of different microfabrication processes involving different materials has been successfully achieved and the operation of the microinterferometer was demonstrated.
With the probing wafer fully operative, the next step concerns its integration in the complete inspection system together with the laser interferometer and the excitation modules. Although a $5 \times 5$ demonstrator has been realized with a fixed pitch, the developed architecture allows its application on a large variety of test wafers by changing the pitch between channels and/or the size of the matrix. Moreover, denser interferometer matrixes could also speed up the measurement time of a whole wafer.

\section{Acknowledgments}

The authors would like to thank the colleagues of the Center for Advanced Micro- and Nano-Optics of the Fraunhofer IOF for their contributions to the fabrication of special parts of the interferometer array wafer, especially Sylke Kleinle, Gilbert Leibeling and Ralf Rosenberger. This work has been realized in the framework of the SMARTIEHS collaborative project, funded under the Grant Agreement 223935 to the 7th Framework of the European Union.

\section{References}

[1] Osten W, Juptner W, Seebacher S and Baumbach T 1999 Qualification of optical measurement techniques for the investigation of material parameters of microcomponents Proc. SPIE 3825 152-64

[2] Gorecki C, Józwik M and Sałbut L 2005 Multifunctional interferometric platform for on-chip testing the micromechanical properties of MEMS/MOEMS J. Microlith. Microfab. Microsyst. 4 1-10

[3] Seebacher S, Osten W, Baumbach T and Juptner W 2001 The determination of material parameters of microcomponents using digital holography Opt. Laser Eng. 36 103-26

[4] Petitgrand S, Yahiaoui R, Danaie K, Bosseboeuf A and Gilles J P 2001 3D measurement of micromechanical devices vibration mode shapes with a stroboscopic interferometric microscope Opt. Laser Eng. 36 77-101

[5] Aswendt P, Hofling R and Hiller K 1999 Testing microcomponents by speckle interferometry Proc. SPIE $3825165-73$ 
[6] Petrov V and Bernhard L 1999 ESPI with holographically stored waves and other innovative ESPI methods used for real-time monitoring of dynamic thermal deformations in a practical industrial environment Proc. SPIE 3824 262-73

[7] Harasaki A, Schmit J and Wyant J C 2000 Improved vertical scanning interferometry Appl. Opt. 39 2107-15

[8] Gastinger K, Haugholt K H, Kujawinska M, Jozwik M, Schaeffel C and Beer S 2009 Optical, mechanical and electro-optical design of an interferometric test station for massive parallel inspection of MEMS and MOEMS Proc. SPIE $738973891 \mathrm{~J}$

[9] http://www.cmicro.com

[10] http://www.melexis.com

[11] Beer S, Zeller P, Blanc N, Lustenberger F and Seitz P 2004 Smart pixels for real-time optical coherence tomography Proc. SPIE 5302 21-32

[12] http://www.schott.com/hometech/english/download/ brochure_borofloat_e.pdf

[13] http://www.microchem.com/products/pmgi.htm

[14] Guenther K H, Wierer P G and Bennett J M 1984 Surface roughness measurements of low-scatter mirrors and roughness standards Appl. Opt. 23 3820-36
[15] Fuchs U, Zeitner U D and Tünnermann A 2006 Hybrid optics for focussing ultra-short laser pulses Opt. Lett. 31 1516-8

[16] Popovic Z, Sprague R and Neville Conell G A 1988 Technique for monolithic fabrication of microlens arrays Appl. Opt. 23 1281-4

[17] Daly D, Stevens R F, Hutley M C and Davies N 1990 The manufacture of microlenses by melting photoresist Meas. Sci. Technol. 1 759-66

[18] http://www.microresist.de/products/ormocers/ ormocomp_en.htm

[19] Dannberg P, Mann G, Wagner L and Bräuer A 2000 Polymer UV-moulding for micro-optical systems and O/E-integration Proc. SPIE 4179 137-45

[20] Radtke D and Zeitner U D 2007 Laser-lithography on non-planar surfaces Opt. Express 15 1167-74

[21] Gale M T 1997 Direct writing of continuous-relief micro-optics Micro-Optics. Elements, Systems and Applications ed H P Herzig (London: Taylor and Francis) pp 87-126

[22] Malitson I H 1965 Interspecimen comparison of the refractive index of fused silica J. Opt. Soc. Am. 55 1205-09 